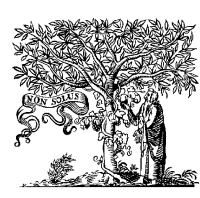

ELSEVIER

Journal of Molecular Catalysis A: Chemical 166 (2001) 47-52

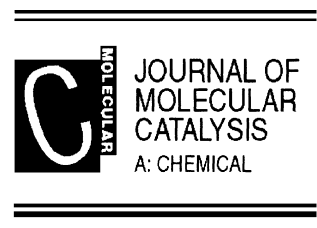

www.elsevier.com/locate/molcata

\title{
The quantum structure of carbon tori
}

\author{
S.A. Bovin*, L.F. Chibotaru, A. Ceulemans \\ Department of Chemistry, Katholieke Universiteit Leuven, Celestijnenlaan 200F, 3001 Heverlee, Belgium
}

Received 4 January 2000; received in revised form 16 March 2000; accepted 22 March 2000

\begin{abstract}
Carbon nanotubes are very promising new materials that fit perfectly well into the miniaturisation of technology, but at the same time show a fascinating quantum structure. When the tube is closed to form a torus the structure acquires discrete molecular characteristics. In the present paper the electronic levels of tori are obtained by a double folding of the Brillouin zone of graphitic carbon. An illustrative example of an hypothetical giant torus with molecular symmetry is fully analysed, with special emphasis on its pictorial representation. (C) 2001 Elsevier Science B.V. All rights reserved.
\end{abstract}

Keywords: Nanotubes; Toroids; Zone folding

\section{Introduction}

Material science is quickly approaching the realisation of molecular objects with toroidal topology. Circular carbon nanotubes have already been reported [1-4] but are probably open coils rather than closed tori. From the theoretical point of view, toroidal quantum structures offer fascinating new perspectives which should further be explored without delay.

As we have described elsewhere [5] the electronic structure of perfect tori made up of carbon hexagons can be obtained in the tight-binding approximation by a double zone folding procedure. In the present paper we apply this method to generate the spectrum of a giant torus, and to analyse its symmetries.

\section{The construction of toroids from the graphene sheet}

A polyhex carbon toroid can be constructed by cutting out a parallellogram from the graphene sheet,

\footnotetext{
* Corresponding author.
}

rolling it up to a tube by joining two opposite edges and then pasting together the top and bottom tubular ends.

Two vectors are introduced to describe this process. The first one is the chiral vector, $\boldsymbol{C}_{\boldsymbol{h}}$, which runs along the circumference of the tube. In the standard hexagonal lattice this vector is described by indices $(n, m)$, as shown in Fig. 1:

$\boldsymbol{C}_{\boldsymbol{h}}=n \boldsymbol{a}_{1}+m \boldsymbol{a}_{2}$

Because of the high symmetry of the honeycomb lattice there are twelve equivalent ways to label a given tube. By convention, only a $30^{\circ}$ wedge is therefore considered so as to have a one-to-one correspondence between $(n, m)$ indices and tubes (cf. Fig. 2) [6]. Within this wedge only two directions result in non-chiral tubes: the armchair direction $(n, n)$ and the zigzag direction $(n, 0)$. Note that for other directions the strip itself, as long as it is flat, has no intrinsic handedness. Chirality only appears when the folding starts, i.e. there are two ways to fold the strip along the chiral vector, forward or backward, which will produce enantiomeric forms. Since we do not at present consider optical activity, we will not pay attention to 


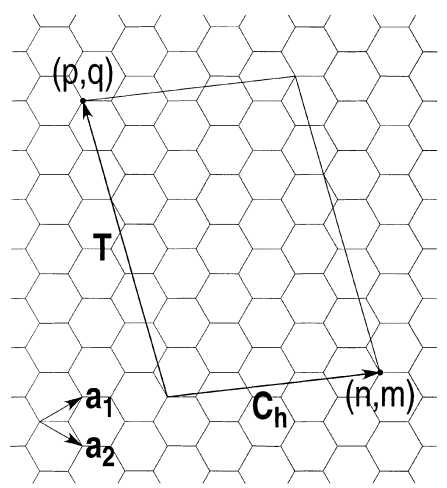

Fig. 1. Definition of the chiral vector $\boldsymbol{C}_{\boldsymbol{h}}$ and the twist vector $\boldsymbol{T}$. This example shows a $(3,2,5,-7)$ torus.

the distinction between antipodes. The second vector is the twist vector $\boldsymbol{T}$. It links atoms on the open tubular ends which will be glued together when the ends are joined. Its indices will be denoted as $(p, q)$ :

$\boldsymbol{T}=p \boldsymbol{a}_{1}+q \boldsymbol{a}_{2}$

The vector $\boldsymbol{T}$ is chosen in the $180^{\circ}$ wedge of the lattice counterclockwise from $\boldsymbol{C}_{\boldsymbol{h}}$ to $-\boldsymbol{C}_{\boldsymbol{h}}$

We thus arrive at a four-parameter toroid label $(n, m, p, q)$ which is in fact redundant and

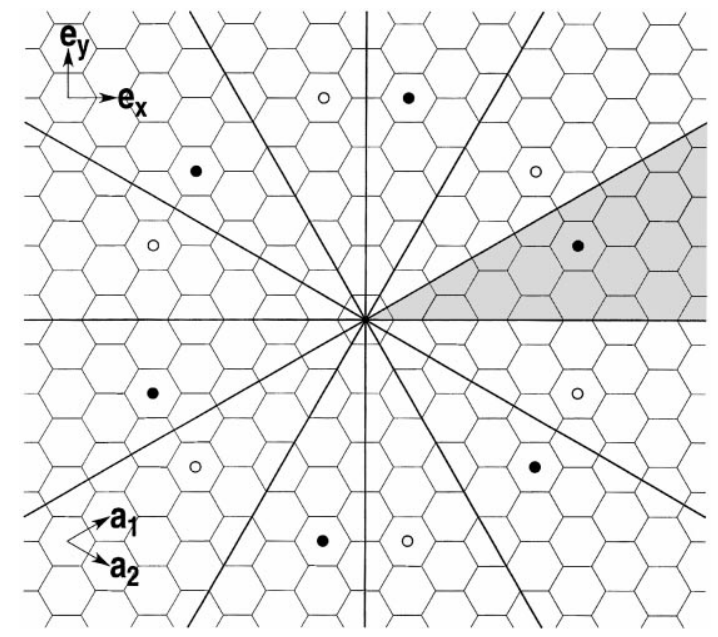

Fig. 2. A given vector can be realised in twelve different ways on the graphene sheet. All solid circles are equivalent under six-fold rotation, while the open circles are the images of the solid ones under two-fold rotations along axis in the plane of the sheet. Only the $30^{\circ}$ gray wedge is considered when defining the chiral vector. non-unique. More rigorous labeling systems, requiring only three indices, have been described $[7,8]$. But for present purposes the $(n, m, p, q)$ system is more practical. The equivalent rigorous code can always be found [5]. From simple geometrical considerations, the number of atoms $N$ in a torus $(n, m, p, q)$ is given by

$$
N=2(m p-n q)
$$

From a topological point of view, the interchange of the chiral and twist vectors generates an equivalent toroid, with the same spectrum. However, from a 3D geometrical point of view these toroids with interchanged chiral and twist vectors are different objects. We therefore, emphasise that in the $(n, m, p, q)$ labeling the $(n, m)$ vector always corresponds to a winding around the tubular ring of the toroid while the $(p, q)$ vector represents a winding around the central hole.

\section{The electronic structure of toroids in the tight-binding approximation}

The $\pi$-electronic structure of toroids arises from the $\pi$-band structure of graphene by a double folding of the Brillouin zone.

Each carbon atom in graphene contributes one electron in a $2 \mathrm{p}_{z}$ orbital projecting perpendicularly from the plane of the sheet. The $\pi$-band corresponds to a bonding and antibonding energy surface [9], described by

$$
\begin{aligned}
E_{k}^{ \pm}= & \pm \gamma_{0} \\
& \times\left\{1+4 \cos \frac{\sqrt{3} k_{x} a}{2} \cos \frac{k_{y} a}{2}+4 \cos ^{2} \frac{k_{y} a}{2}\right\}^{1 / 2}
\end{aligned}
$$

with $\gamma_{0}$ the hopping integral; $a=\left|a_{1}\right|=\sqrt{3} a_{C-C}=$ $\sqrt{3} \times 1.42 \AA$ is the lattice constant and $k_{x}$ and $k_{y}$ are cartesian components of the wave vector. The first Brillouin zone is a regular hexagon (cf. Fig. 3) [10].

The folding of the zone occurs when cyclic conditions are imposed which correspond to the rolling up of the sheet and the closing of the tube. The rolling up of the sheet along the chiral vector constitutes the first 


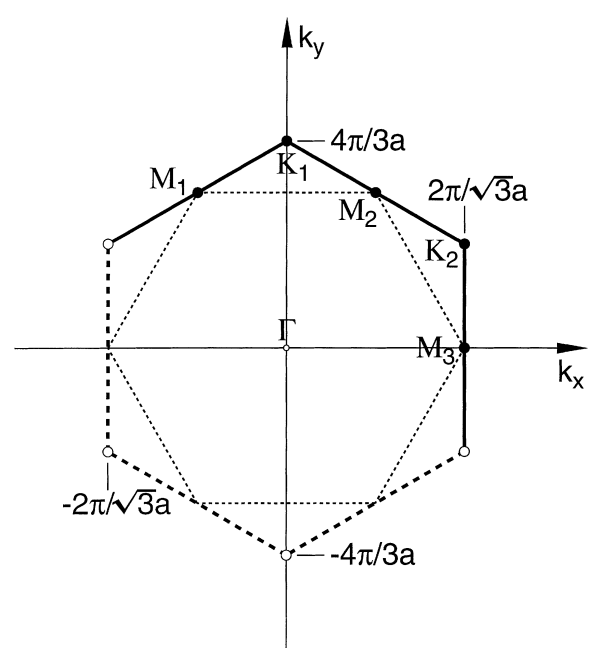

Fig. 3. The first Brillouin zone of the graphene sheet. The solid heavy lines indicate borders that belong to the half-open zone, heavy dashed lines belong to the neighbouring zones. Of the six vertices, only the two K-points belong to the zone. Also indicated are the M-points, the $\Gamma$-point and the line $E(\boldsymbol{k})= \pm 1$ (the dotted line).

zone folding and reduces the Brillouin zone to a set of lines with $k$-values that obey the cyclic condition

$\boldsymbol{C}_{\boldsymbol{h}} \cdot \boldsymbol{k}=2 \pi l_{n}$

where $l_{n}$ is an integer, the nanotube line index. These nanotube lines are perpendicular to the direction of the chiral vector in reciprocal space.

The second folding corresponds to the cyclic condition for the twist vector:

$\boldsymbol{T} \cdot \boldsymbol{k}=2 \pi l_{t}$

where $l_{t}$ is an integer torus line index. The torus lines are perpendicular to the direction of the twist vector in reciprocal space.

The allowed $\pi$-states must obey both cyclic conditions and thus will coincide with the intersection points of nanotube and torus lines. The allowed $k$-points can easily be expressed as functions of $(n, m, p, q)$ and $\left(l_{n}, l_{t}\right)$ :

$\begin{aligned} k_{x} & =\frac{2 \pi}{\sqrt{3} a} \frac{l_{t}(m-n)+l_{n}(p-q)}{m p-n q} \\ k_{y} & =\frac{2 \pi}{a} \frac{l_{t}(n+m)-l_{n}(p+q)}{m p-n q}\end{aligned}$
These equations describe a discrete set of allowed points, with cardinal number $N / 2$. Since for each $k$-point there is a bonding and an antibonding level the total number of states is indeed equal to the number of carbon atoms $N$.

For some values of the $(n, m, p, q)$ parameter set the set of allowed $k$-points may contain special symmetry points of the first Brillouin zone, denoted as the trigonal K-points at zero energy and the digonal M-points at energy $\pm \gamma_{0}$. The central $\Gamma$-point, which has hexagonal symmetry, will always be allowed. It corresponds to the total bonding and antibonding combinations with $\varepsilon_{\Gamma}= \pm 3 \gamma_{0}$. The presence or absence of the K-points in the toroidal eigenvectors is of primary importance. If the K-points are present the occupied and empty bands are touching like in a zero bandgap conductor, while if absent there is an insulating gap between the valence and conduction bands. It can easily be shown that the sufficient condition for presence of the K-points in a torus band is the simultaneous divisibility of $(n-m)$ and $(p-q)$ by 3 .

\section{Analysis of a giant torus}

The coiled samples reported by Martel et al. [4] have average ring diameters of $600-800 \mathrm{~nm}$ and a tubular cross section with a width of approximately $1.4 \mathrm{~nm}$. The latter diameter is of the order of magnitude of fullerene diameters.

It can be estimated that such coils will consist of several hundreds of thousands of atoms. In the present example we will consider for practical reasons a torus which is much smaller but already gives an impression of a giant molecular torus. It should be kept in mind that the interatomic strain in a torus of this size is still too large to maintain a perfectly circular shape [11]. Spontaneous kinks are likely to be formed unless pentagonal and heptagonal defects are introduced. Fig. 4 provides a plot of an ideal circular polyhex torus of type $(10,4,48,-64)$ with $N=1664$ atoms. The ring diameter $D$ and tubular diameter $d$ are given by

$D=\frac{T \cos (\beta-\pi / 2)}{\pi} \approx 45.5 \AA$

$d=\frac{C_{h}}{\pi} \approx 10 \AA$ 




Fig. 4. A view of a large torus $(10,4,48,-64)$ with $D_{16}$ symmetry.

with $\beta$ the angle between $\boldsymbol{C}_{\boldsymbol{h}}$ and $\boldsymbol{T}$ in the strip on the graphite sheet [5]. In these expressions $C_{h}$ and $T$ are the lengths of $\boldsymbol{C}_{\boldsymbol{h}}$ and $\boldsymbol{T}$ and are given by

$$
\begin{aligned}
& C_{h}=a\left(n^{2}+m^{2}+n m\right)^{1 / 2} \\
& T=a\left(p^{2}+q^{2}+p q\right)^{1 / 2}
\end{aligned}
$$

An analysis of the point group symmetry of this torus is based on the subgroup structure of the cylindrical group $D_{\infty h}$. If the chiral vector does not point along the armchair or zigzag directions, the corresponding nanotube will be chiral, and so will be the final torus. For such a chiral structure no improper symmetry elements are allowed, leaving only $C_{v}$ or

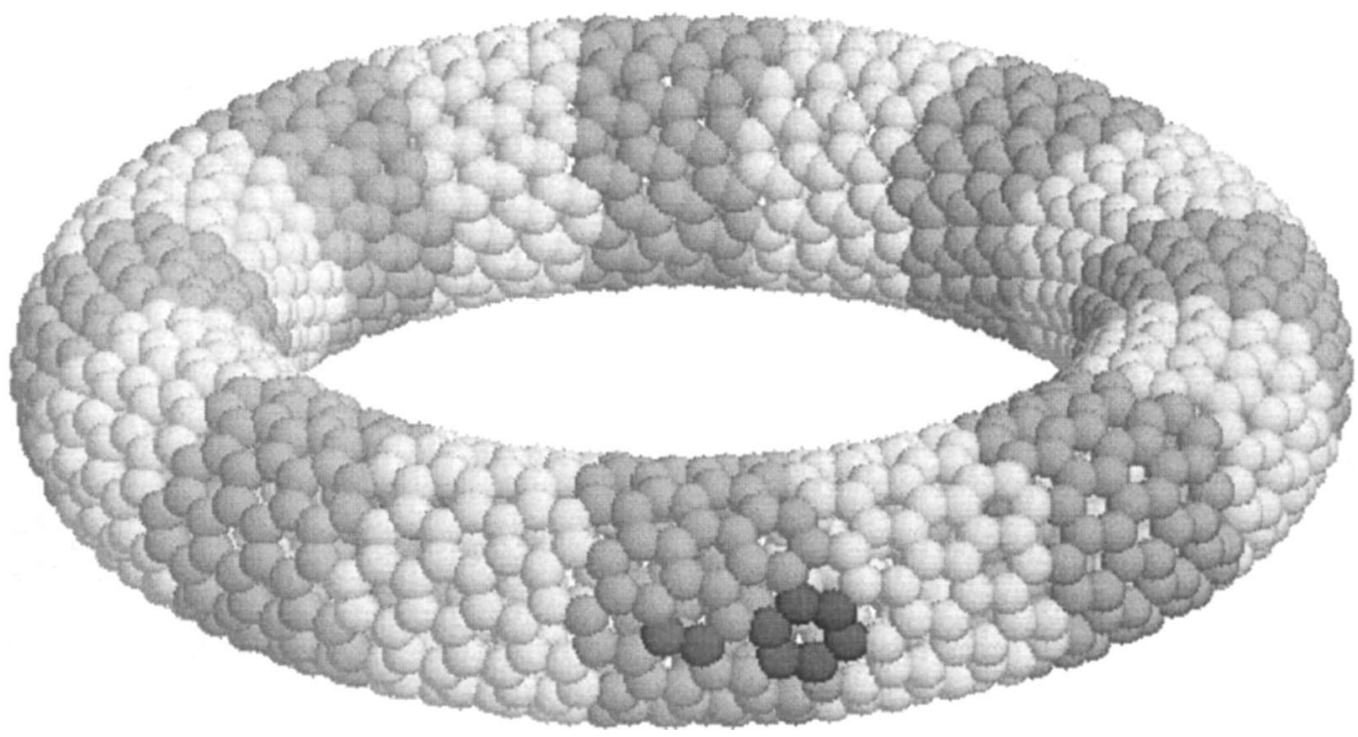

Fig. 5. Model of torus $(10,4,48,-64)$ highlighting the $D_{16}$ symmetry. Alternating rotational units are shaded darker. The $C_{2}$ axes pass through the centre of the bond between the two dark atoms and through the centre of the dark hexagon. 


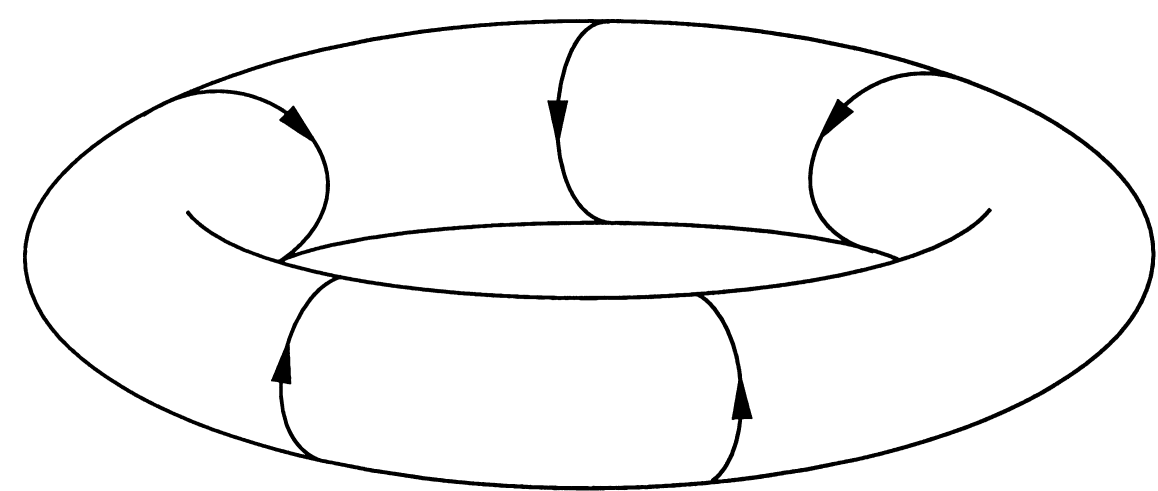

Fig. 6. Anapole whirling motion around the tubule forming the toroid. This motion does not destroy the principal rotational axis.

$D_{v}$ as possible point groups. Such is the case for the present torus.

A rotation by an angle $\alpha=2 \pi / \nu$ about an axis through the centre of the torus corresponds on the unfolded strip to a translation over a distance $T / v$ along the twist vector. This will be a symmetry operation if $v$ is a common factor of $p$ and $q$. Hence in general we may conclude that a torus $(n, m, p, q)$ has a prin- cipal $C_{v}$ axis, where $v$ is the largest common divisor of $p$ and $q$. As an example, for the torus in Fig. 4 the largest common factor of $p=48$ and $q=-64$ equals 16 and we indeed have a $C_{16}$ rotation axis through the centre (cf. Fig. 5). This conclusion is independent of the actual revolution of the tubular frame around its spine. This whirling motion, which is represented in Fig. 6, transforms in cylindrical symmetry as $\Sigma_{u}^{+}$,

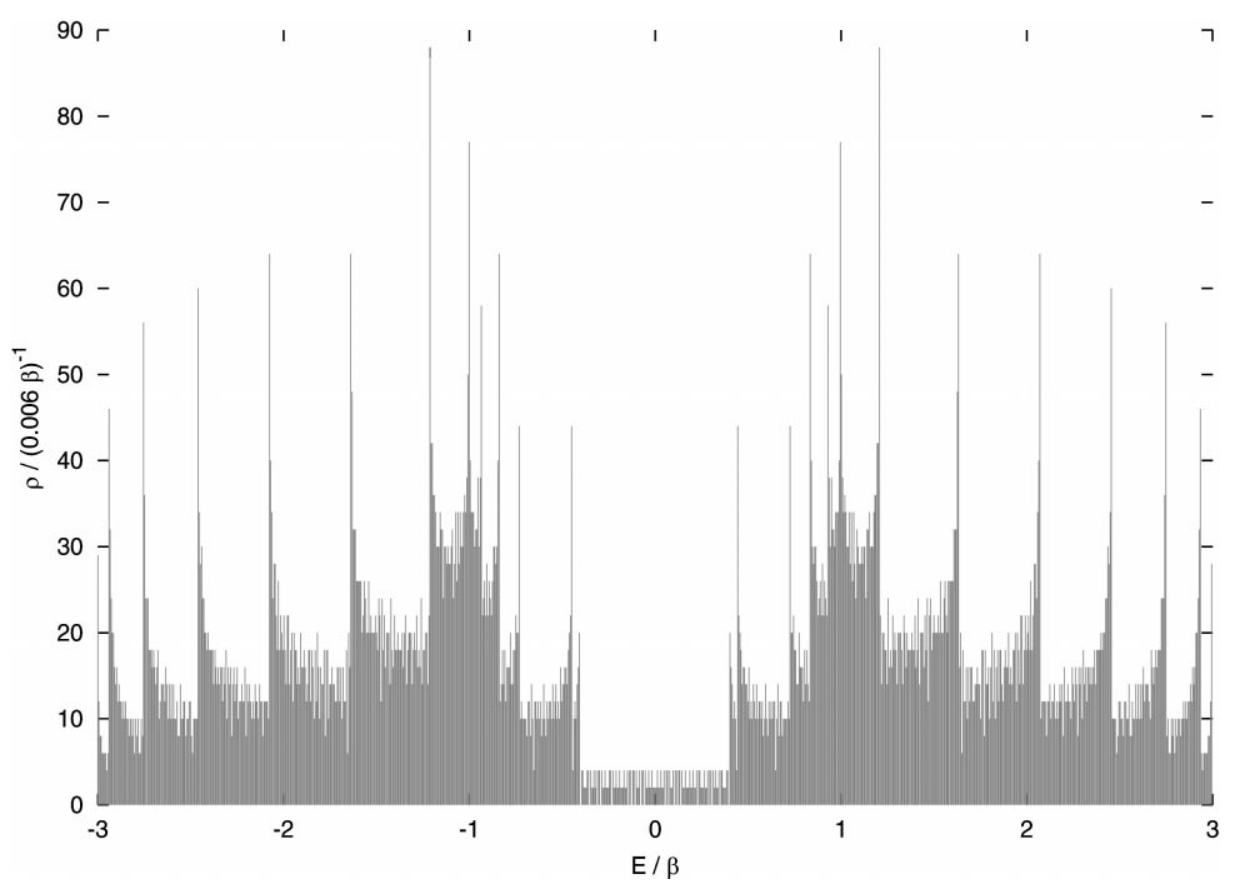

Fig. 7. Density of States for a giant torus $(10,4,480,-640)$. 
and is referred to as the molecular anapole mode [12]. Because of its symmetry it is able to destroy the horizontal symmetry plane and the two-fold rotation axis perpendicular to the cylindrical axis, but not the vertical planes or principal rotation elements.

Perpendicular to its principal $C_{v}$ symmetry a chiral torus can have additional $C_{2}$ axes in its equatorial plane if centres of bonds or hexagonal faces are lying precisely in the equatorial plane. This thus will depend on the actual $3 \mathrm{D}$ realisation of the torus and cannot be decided from the unfolded strip. Knowing the positions of the atoms, such special points can easily be found. As an example, the giant torus in Fig. 4 has equatorial $C_{2}$ axes and thus has point group symmetry $D_{16}$ (cf. Fig. 5).

We also have determined the electronic energy levels of giant toroids using the double zone folding procedure. The results for a torus which is ten times as big as the one of Fig. 4 are represented in a Density of States plot in Fig. 7. This diagram is extremely similar to DOS plots for nanotubes (see e.g. [13]).

One easily recognises the bipartite character of the energy bands of the graphite sheet. Further note the clear resonances or Van Hove singularities which correspond to the nanotube lines. The parent tube of our choice is of the metallic type $(n-m$ is divisible by 3 ), which gives rise to the typical zero-gap Fermi region. However, the difference between the twist vector parameters, $p-q$, is not divisible by 3 . As a result there will be a very tiny 'toroidal' bandgap of approximately $0.63 \times 10^{-2} \gamma_{0}$.

\section{Conclusion}

The electronic structure of carbon toroids can be obtained by a straightforward extension of the nanotube zone folding procedure along the direction of the tube. For giant tori the resulting Density of States diagram is very similar to the diagram of infinite tubes.

\section{Acknowledgements}

This research was supported by a grant from the Belgian Government (Programmatie van het Wetenschapsbeleid).

\section{References}

[1] J. Liu, H. Dai, J.H. Hafner, D.T. Colbert, R.E. Smalley, S.J. Tans, C. Dekker, Nature (London) 385 (1997) 780.

[2] T. Vossmeyer, S.-W. Chang, W.M. Gelbart, J.R. Heath, Adv. Mater. 10 (1998) 351.

[3] R. Martel, H.R. Shea, P. Avouris, Nature (London) 398 (1999) 299.

[4] R. Martel, H.R. Shea, P. Avouris, J. Phys. Chem. B 103 (1999) 7551.

[5] A. Ceulemans, L.F. Chibotaru, S.A. Bovin, P.W. Fowler, J. Chem. Phys. 112 (2000) 4271.

[6] M.S. Dresselhaus, G. Dresselhaus, P.C. Eklund, Science of Fullerenes and Carbon Nanotubes, Academic Press, San Diego, 1995.

[7] E.C. Kirby, R.B. Mallion, P. Pollak, J. Chem. Soc., Faraday Trans. 98 (1993) 1945.

[8] S. Negami, Discrete Math. 44 (1983) 161.

[9] P.R. Wallace, Phys. Rev. 71 (1947) 622.

[10] S.L. Altmann, Band Theory of Solids: An Introduction from the Point of View of Symmetry, Clarendon, Oxford, 1991.

[11] V. Meunier, Ph. Lambin, A.A. Lucas, Phys. Rev. B 57 (1998) 14886.

[12] A. Ceulemans, L.F. Chibotaru, P.W. Fowler, Phys. Rev. Lett. 80 (1998) 1861.

[13] M.S. Dresselhaus, G. Dresselhaus, P.C. Eklund, R. Saito, in: J. Shinar, Z.V. Vardeny, Z.H. Kafafi (Eds.), Optical and Electronic Properties of Fullerenes and Fullerene-Based Materials, Marcel Dekker, New York, 2000, Chapter 8, pp. 217-260. 